\title{
HELIUM RUNAWAYS IN WHITE DWARFS
}

\author{
Ronald.E. Taam \\ Department of Physics and Astronomy \\ Northwestern University \\ Evanston, Illinois 60201
}

Received: 1979 August 8;

(NASA-CR-162694) HELIUN RONATAYS IN WHITE

N80- 16986

DRARFS (Northwestern Univ.) $22 \mathrm{p}$

HC A 02/AL AO 1

CscI, $03 \mathrm{~A}$

Juclas

G3/89 11620

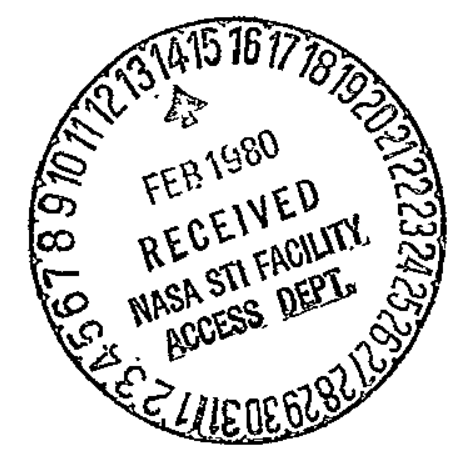


ABSTRACT

The long term evolution of an accreting carbon white dwarf $\left(\mathrm{M}=0.5 \mathrm{M}_{\theta}\right)$ has been studied from the onset of accretion to the ignition of helium. The variations in the detalls of the helium shell flash have been examined with respect to variations in mass accretion rate. For intermediate rates $\left(10^{-9}-10^{-8} \mathrm{M}_{0} \mathrm{Yr}^{-1}\right)$ the helium flash is potentially explosive whereas for high rates ( $5 \times 10^{-8} \mathrm{M}_{\theta} \mathrm{yr}^{-1}$ ) the shell flash is relatively weak. The results are discussed in the context of the long term evolution of novae. Subject headings: stars: accretion - stars: evolution - stars: interiors - stars: novae - stars: white dwarfs 


\section{INTRODUCTION}

It Is generally accepted that the cause of the nova outburst is attributable to a thermonuclear runaway in the hydrogen-rich layers of an accreting white dwarf (cf. Starrfield, Sparks, and Truran 1974a; Prialnik, Shara, and Shaviv 1978). In this model, the hydrogen-rich matter is accumulated until critical temperatures and densi..ties - are $\cdot$ reacheds whereupon the accreted material wis, burned. The rapid release of nuclear energy heats the envelope and eventually leads to its ejection. The strength of the outburst is primarily determined by the abundance of the CNO nuclei and the mass of the white dwarf. The long term evolutionary behavior of the white dwarf has recentIy been the subject of several investigations. Two groups of workers (Paczynski and Zytkow 1978; Sion, Acierno, and Tomczyic 1979) have followed a number of hydrogen shell flashes for a wide range of white dwarf masses and mass accretion rates. The results of these studies, taken as an aggregate, delineate the regime for which hydrogen burning is unstable. In this regime, they show that the interflash perlod is a strongly decreasing function of both white dwarf mass and mass accretion rate. Work on the underlying helium-rich layer has also received some attention. Webbink, Truran, and Gallagher (1978) have proposed that helium runaways may be an important ingredient in determining the overall strength of the nova outburst. Since the repeated hydrogen shell flashes lead to the accumulation of an underlying helfum-rich layer, they suggest that hellum flashes may also be recurrent, but on a longer timescale. They point out that the convective mixing which develops during the flash 
could provide the enhanced CNO abundances in a natural manner (see also, Taam and Faulkner 1975). Nomoto and Sugimoto (1977) have also focused upon the long term consequences of the helium-rich matter. By neglecting the thermal effects of the hydrogen shell flashes on the underlying layers, they investigated the development of the helium runaway in a helium white dwarf. They found that the character of the nuclear flash was extremely sensitive to the efficiency of compressional heating and, hence, to the mass accretion rate. For rapid mass accretion ( $\dot{\mathrm{M}}<4 \times 10^{-\overline{8}_{\mathrm{M}}} \mathrm{yr}^{-1}$ ), a shell flash resulted which could lead to the mixing. hypotheslzed by Webbink et al. (1978) and for slow mass accretion $\left(\dot{M} \leqslant 2 \times 10^{-8} \mathrm{M}_{\theta} \mathrm{yx}^{-1}\right.$ ) a central Elash resulted leading to a Type I supernova exploston and to the complete disruption of the white dwarf (see also, Mazurek 1973). Although helium white dwarfs in nova binary systems cannot be excluded, there is strong observational and theoretical evidence which suggests that carbon white dwarfs are more appropriate (see below).

Accordingly, it is the purpose of this investigation to examine the detafls of the helium flash in a carbon whtte dwarf for a range of mass accretion rates. In SII, we formulate the problem and outline our basic assumptions. The numerical results are presented in \$III and in \$IV we conclude with a discussion of the implications of our results for the long term evolution of novae.

\section{FORMULATION AND ASSUMPTIONS}

The accretion of matter is considered in the spherically symetric approximation. Helfum-rich materlal $(X=0, Y=.98, Z=.02)$ is added, at a constant rate, to the stellar surface in a quiescent manier with an initial specific entropy equal to that of the surface. The constitutive relations and physical data are similar to those described in 
Taam (1979) and w111 not be presented here. We assume an Initial white dwarf mass of $0.5 M_{0}$. The results of Starrfleld, Sparks, and Truran (1974b) and Nartai, Nomoto, and Sugimoto (1977), indicate that nova explosions are weak or non-existent for white dwarfs of mass less than about $0.5 \mathrm{M}_{\theta}$. This difficulty stems from the small surface gravities typical of low mass white dwarfs which leads to a low degree of electron degeneracy in the hydrogen burning shell and, therefore, to a weak hydrogen shell flash. Since little or no material is efected from the white dwarf surface, a constant mass accretion rate should be applicable. Over a long timescale (long compared with the interflash pertod of the hydrogen shell flashes), a significant amount. of helium will, therefore, accumulate on the white dwarf. These shell flashes, although important for the short term evolution of the nova, are not explicitly included in our study. For the mass accretion rates $\left(10^{-9}-10^{-8} \mathrm{M}_{0} \mathrm{yr}^{-1}\right.$, see Warner 1976) which are thought to be relevant to nova binaries, an examination of the energy transport from the hydrogen burning shell to the white dwarf core reveals that the depth to which energy is conducted is mintmal (Taam 1977). That is, heating of the underlylng (helium-rich) layer Is ineffective as nearly all the nuclear energy is transported to the surface. As such, the long term effects of the hydrogen burning shell on the thermal structure of the deep envelope layers are, in this case, unimportant. On the other hand, the lack of immediate adjustment to the newly"accreted material can lead to heating, in the interior. For mass accretion rates in excess of about $10^{-9} \mathrm{M}_{\theta} \mathrm{yr}^{-1}$, compressional heatIng will dominate and, hence, the thermal structure will be governed by the dfsparity between the mass accretion and thermal readjustment 
tImescales. The shorter the mass accretion timescale, the more near1y adlabatic will be the compressional heating. In such cases, the effects of the hydrogen burning layer may be approximately neglected and the problem of the long term evolution of a white dwarf accreting hydrogen reduces to one in which helium may be directly accreted.

As alluded to earlier, a principal conclusion of the study by Starrfleld, Sparks, and Truran (1974a) was the need for enhanced CNO abundances in order to reproduce the gross features of fast nova outbursts. This requirement, naturally, led these authors to suggest that the outburst occurs on a carbon white dwarf. It is made plaustble by the fact that the masses of white dwarfs in nova binaries, as. inferred from observational data, are large $\left(M-\operatorname{IM}_{\theta}\right.$, Robinson 1976) coupled with the theoretical viewpoint that the cores of the wore massive white dwarfs should be composed of carbon or material of greater atomic weight. The results of single star evolution (if applicable to binaries) indicate that stars more massive than $0.45 \mathrm{M}_{\theta}^{\circ}$ ignite helium prior to becomIng white dwarfs (Webbink 1975). Thus, the Interior of more massive stars should consist of the products of helium burning, 1.e. carbon and oxygen. It should be noted, however, that more massive helfum white dwarfs $\left(M>0.45 M_{\theta}\right)$ could be built up by mass accretion in binaries. Normally, the production of a white dwarf requires a previous giant stage and, therefore, a relatively wide initial separation between. the comporients of the original binary system. Since nova binarles are found at short perlods ( several hours), the manner in which such systems are formed remains obscure. Evolutionary processes by which this transformation may have taken place are discussed by Ritter (1976) and Paczynsk1 (1976). Since the detalls of the mechanisms have yet to 
be quantified, we adopt the point of view that we may Investigate the evolution of a white dwarf in a short period system without regard to the issue of its formation.

\section{III. - RESULTS}

The evolution of a carbon white dwarf accreting helfum-rich matter was followed utilizing the stellar evolution code developed and described by by Eggleton $(1971,1972)$. ThIs hydrostatic code utilizes a non-Lagranglan mesh, which includes the entire envelope, and is well suited for investigations of mass accretion (or mass 1oss). The initial model is characterized by an effective temperature $\mathrm{T}_{e}=1.23 \times 10^{4} \mathrm{~K}$, a luminosity $\mathrm{L}_{\mathrm{s}}=4.31 \times 10^{-3} \mathrm{~L}_{\theta}$, and a central temperature $T_{c}=1.8 \times 10^{7} \mathrm{~K}$. Three evolutionary sequences were computed, each distinguished by the rate of mass accretion $(M=.1,1$, $\left.5 \times 10^{-8} \mathrm{M}_{\theta} \mathrm{yr}^{-1}\right)$..

For these rates; compressional heating effects determine the course of the evolution. As mass is accreted, the inner layers contract and the gas is compressed, thereby releasing gravitattonal energy in the process. Since the mass accretion timescales are shorter than the thermal readjustment timescales in the interior and the deep envelope layers, this energy cannot be radiated quickly enough and the interfor temperatures rise. In fact, compressional effects significantly affect the central regions of the white dwarf as well. This is in contrast to the static interior conditions found in the short term evolution because the amount of mass accumulated $\left(\Delta M>0.1 M_{\theta}\right)$, here, is not negligible, compared to the total mass of the white dwarf. In the following, we present the results for the cases of rapid, intermediate, and slow mass accretion. 


\section{(1) Rapid Mass Accretion}

A high rate of mass accretion $\left(\mathrm{M}=5 \times 10^{-8} \mathrm{M}_{\theta} \mathrm{yI}^{-1}\right)$ is considered in order to maximize the effects of compressional heating. The numerIcal results of the evolutionary calculations are summarized in Table 1 and illustrated in the temperature-density diagram in Fig. 1 . It is clear that the thermal structure of the entire white dwarf is affected. In particular, an off-centered temperature maximum forms in the vicinity of the He-C composition interface (corresponding to the region of partial degeneracy) where the gravitational energy release associated with the compression is effectively localized. Note that the mass point corresponding to the temperature maximum does not colncide with the base of the accreted layers (see Fig. 1). For the high degree of electron degeneracy $(\psi \geq 100$, where $\psi$ is the electron degeneracy parameter) characteristic of the interior, the high efficiency of electron conduction Inhibits the growth of large temperature differences. The more effective heating in the partialiy degenerate regions is made evident upon examination of Fig. 1 where it is seen that the rlse in shell temperature, $T_{m}$, increases from $5.1 \times 10^{7} \mathrm{~K}$ to $7.1 \times 10^{7} \mathrm{~K}$ in $7.9 \times 10^{5} \mathrm{yrs}$. as compared to the rise in central temperature, $T_{c}$, from $1.9 \times 10^{7} \mathrm{~K}$ to $2.1 \mathrm{x}$ $10^{7} \mathrm{~K}$ (see Table 1 ). After $0.112 \mathrm{M}_{\Theta}$ has been accumulated $\left(t \sim 2.24 \times 10^{6} \mathrm{yrs}\right.$ ), the physical conditions are such that $C_{\mathrm{m}}=1.7 \times 10^{5} \mathrm{gm} \mathrm{cm}$ and $\mathrm{T}_{\mathrm{m}}=8.8 \mathrm{x}$ $10^{7} \mathrm{~K}$ ) helium is ignited. Because of the strong temperature dependence of the helium energy generation rates and the moderate degeneracy of the electron gas in the burning shell $(\psi \sim 7)$, a degenerate shell flash ensues. Within $1.6 \times 10^{5}$ yrs., a convective region forms immediately, above. the nuclear burning shell. At this stage, the shell is characterized 
by a temperature of $1.1 \times 10^{8} \mathrm{k}$, a density of $2.05 \times 10^{5} \mathrm{gm}^{\mathrm{cm}}{ }^{-3}$, and a nuclear luminosity of $2.9 \times 10^{3} \mathrm{~L}_{\theta^{*}}$. It takes an additional $40 \mathrm{grs}$. to reach $1.8 \times 10^{8} \mathrm{~K}$ and $6.9 \times 10^{8} \mathrm{~L}_{\theta}$ by which point the degeneracy of the electron gas is decreasing. At this time, convection is inefficient as superadiabatic temperature gradients were required to transport the flux. The mass in the growing convective shell increased to $0.08 \mathrm{M}_{\theta}$, and the densities at its outer boundary decreased to $4 \times 10^{3} \mathrm{gm}^{-3}$. The subsequent evolution of the helium burning shell is, now, governed by the nondegenerate shell flash instability. In the next $5.6 \times 10^{4}$ seconds, the density, temperature, and nuclear luminosity in the shell reached $10^{5} \mathrm{gm} \mathrm{cm} \mathrm{cm}^{-3}, 2.5 \times 10^{8} \mathrm{~K}$, and $7.3 \times 10^{10} \mathrm{~L}_{\theta}$, respectịvely. As a result of the expansion of the outer core (i.e. He-C composition boundary), the central regions expanded and cooled adiabatically (see Table 1). At all phases of the thermial flash, the star remained in hydrostatic equilibrium. The calculations were discontinued when it became apparent that the progress of the shell flash was qualitatively similar to that found ${ }^{-1}$ in case $A$ of -Nomoto and Sugimoto (1977). Although Nomoto and Sugimoto (1977) considered a $0.4 \mathrm{M}_{\theta}$ helium white dwarf, a comparison of Table $\ddot{1}$ with their Tables 2 and 3 shows reasonably good agreement.

\section{(i1) Intermediate Mass Accretion}

For this evolutionary sequence, a mass accretion rate of $10^{-8} \mathrm{M}_{\theta} \mathrm{yr}^{-1}$ Is constdered. Because of the longer mass accretion timescale, the compressional heating is less adiabatic, and the energy is not as effective1y localized as found in case (i). The results of our computations to the ignition of hellm are presented In Table 2. Although the compression rate considered, here, is lower, the central temperatures exceed those found to develop in case (1). This is attributable to the greater amount of mass which is accumulated prfor to the ignition of helfum 
$\left(0.617 \mathrm{M}_{\theta}\right.$ as compared with $0.12 \mathrm{M}_{\theta}$ for case (i)). It is clear from the temperature-density diagram (FIg. 2) and from Table 2 that after $0.55 \mathrm{M}_{\theta}$ has been accreted, the heat transport in the interior results in an isothermal distribution. Helium ignition occurs at a density of $\rho_{\mathrm{m}}=1.45 \times 10^{7} \mathrm{gm} \mathrm{cm}^{-3}$ and a temperature of $\mathrm{T}_{\mathrm{m}}=7.35 \times 10^{7} \mathrm{~K}$. Because of the high degree of electron degeneracy $(\psi \sim 100)$, this situation becomes potentially explosive. Since the pressure is insensitive to varlations in the temperature, the nuclear burning will occur under hydrostatic conditions until the heating timescale, 'nuc' is reduced to values comparable to the hydrodynamic response timescale, $\tau_{\text {ff }}$ (see below). That is, under these circumstances, stellar expansion and adiabatic cooling cannot control the nuclear reactions (cf. Arnett 1969). As a result, the shell temperatures rise rapidly. For instance, the maximum shell temperatures increased from $3.3 \times 10^{8} \mathrm{~K}$ to $6.24 \times 10^{8} \mathrm{~K}$ in 0.002 seconds as compared to the rise in temperature from $1.21 \times 10^{8} \mathrm{~K}$ to $1.57 \times 10^{8} \mathrm{~K}$ in 6.55 $\times 10^{3}$ seconds. Even at the high densitiès $\left(\rho-10^{7} \mathrm{gm}^{-3}\right)$ characteristic of the nuclear burning region, the convection could no longer be considered efficient. Superadiabatic temperature gradients $\left(\nabla-\nabla_{\text {ad }}\right)$ of about 4 were required to transport the energy. These steep temperature gradients necessarily confined the rapid burning to a narrow shell (the energy generation rates decreased. by more than 6 orders of magnitude for a 30\% decrease in pressure). It can be seen from Tabie 2 that (i) the characteristlc timescale for the temperature rise in the burning shell due to nuclear reactions, denoted as $\tau_{\text {nuc' }}$, becomes increasingly short with greater temperatures and (ii) the expansion of the she1l is small $\left(\Delta \log \rho_{m}=0.156\right)$. A comparison of $\tau_{\text {nuc }}$ with $\tau_{f f}$, the free fall timescale which we take to be approximately equal to the adiabatic expansion 
timescale, at $\log T_{\text {m }}=8.795$ yields $\tau_{\text {nuc }} / \tau_{\text {ff }}=0.01$. Under such conditions, significant burning is possible prlor to the hydrodynamic response which must ultimately govern the further evolution of the star. At this point, the calculation was discontinued as hydrodynamic effects, which were not included, were starting to become important. The thermonuclear flash develops on such a short time scale - that little energy is. conducted to.the central regions. It is expected that during the hydrodynamic phase; expansion and adlabatic cooling of the central regions will result. For the physical conditions in the helium burning shell found here, ft is posstble that a detonation wave will form offcenter and propagate through the heliumrich materlal. This is in contrast to the investigations of Mazurek (1973) and Nomoto and Sugimoto (1977) where a detonation wave formed at the center of a helium white dwarf.

\section{(111) Slow Mass Accretion}

In order to exaggerate the differences between the results of sequences (i) and ( $i i)$, a mass accretion rate of $10^{-9} \mathrm{M}_{\theta} \mathrm{gr}^{-1}$ was chosen. The evolutionary changes in the structure, are exhibited in the temperature-density diagram (Fig. 3) for the phases prior to helfum ignition. During the initial stages of accretion, it is seen that the temperature distribution becomes non-monotonic, but after this transient phase, the white dwarf evolves without a temperature inverston. Because the accretion timescale is 10 times longer than the intermediate case, the compressional heating is not as effective and the onset of the degenerate flash is delayed to much greater densities ( $\rho-3 \times 10^{8} \mathrm{gm} \mathrm{cm}^{-3}$, see Table 3 ). 
Because of the strong electron screening factor, the helfum burning rates were enhanced by a factor of $10^{8}$ ( at $\mathrm{T}=5.3 \times 10^{7} \mathrm{~K}$ ). Since. the densittes in the shell are more than an order of magnitude greater than found in case (ii), the time scale for the development of the flash, from a given temperature, would be greatly reduced. Helfum Ignition was found to occur after $0.88 \mathrm{M}_{\odot}$ had been accumulated. The calculations were followed to the onset of shell helium burning and not through the degenerate shell flash stage as the thermal fevolution is expected to be simflar in kind to that calculated for case (ii).

\section{CONCLUSION}

Thermonuclear runaways inftiated by the accretion of helfum-rich matter onto a carbon white dwarf of $0.5 \mathrm{M}_{\ominus}$ have been investigated to approximate the long term evolution of novae. For the range of mass accretion rates studied $\left(\dot{M}=0.1-5 \times 10^{-8} \mathrm{M}_{\Theta} \mathrm{yr}^{-1}\right)$ compressional heating effects were found to be significant in rafsing the interior temperatures to values appropriate for the ignition of helium. The effectiveness of this heating dictated the subșequent behavior of the helium shell flash. Generally; the lower the rate of compression, the greater the strength of the shell flash as helfum ignttion is delayed until a greater density (electron degeneracy) is reached. For $\dot{\mathrm{M}}=5 \times 10^{-8} \mathrm{M}_{\Theta} \mathrm{yr}^{-1}$. the flash proceeds under hydrostatlc conditions, whereas for $\dot{\mathrm{M}} \sim 0.1-1 \times 10^{-8} \mathrm{M}_{\mathrm{O}} \mathrm{yr}^{-1}$ the helfum may detonate. In the former case, the extensive convection zone formed exterior to the main energy generating region extended to densities $\left(\rho-4 \times 10^{3} \mathrm{gm} \mathrm{cm}^{-3}\right)$ which may lead to the mixing of carbon fnto the overlying hydrogen rtch layers (see also, Webbink et al. 1978). Th1s mechanism could provide the CNO enhaneement required for fast 
nova outbursts (cf. Starrfleld, Sparks, and Truran 1974a). The hydrogen shell flash immediately following the helium runaway is expected to be most rapid and the strength of each succeeding hydrogen flash to be less intense as the abundances of the CNO nuclei are reduced. A . sṭmilar progression of hydrogen flashes would be expected after each $\therefore$ succeeding helium runaway $\left(\tau-\cdots 2: 4 \times 10^{6} \cdot \mathrm{yrs}\right) \cdot{ }^{* F}$ or $\mathrm{H}=10^{-8} \mathrm{M}_{0} \mathrm{Yr}{ }^{-1}$, the complete combustion of all the helium matter is likely. The energy $y \pm e l d$ would be of the order of $10^{51}$ ergs which is greater than the binding energy of the helium matter by about a factor of 4 . If the detonation wave forms and propagates to the surface, the result could be a supernova explosion. Since the time scale for the evolution is short ( $\left.\tau \leqslant 10^{4} \mathrm{sec}\right)$ Iittle heat is conducted into the central regions, and it is quite possible that the carbon white dwarf core will not particlpate in the evolution, and therefore, remain intact. For the lowest mass accretion rate studied $\left(\bar{M}=10^{-9} \mathrm{M}_{\Theta} \mathrm{yr}^{-1}\right)$, the Iiklihood of the formation of a detonation wave is reduced as the internal energy of the degenerate electrons at the burning shell is comparable to the nuclear energy release. Only a fraction of the envelope may be burnt, perhaps resulting in a less energetic event (a nova). Since compressional heating is much less adjabatic for lower mass accretion rates ( $\dot{\mathrm{M}} \leqslant 10^{-9} \mathrm{M}_{\ominus} \mathrm{yr}^{-1}$ ), helium burning in the white dwarf interior may be avolded in such cases. On the other hand, energy transport from the hydrogen burning shell is non-negligible and it is possible that helium burning inftiated by the hydrogen shell flash will develop in the outer surface layers (Taam and Faulkner 1975, Taam 1979). Investigations along these lines are clearly needed. 
Finally we point out that mass ejection during the hydrogen shell flash phase occurs for high mass white dwarfs even with normal-CNo abundances (Sparks, Starrfleld, and Truran 1978, Prialnik, Shara, and Shaviv 1978). Here, radiation pressure plays the major role in ejecting the matter. Our results, which have been derived assuming a constant mass accretion rate, should still be, applicable as, long as a time averaged rate is 'used.

This research has been supported by NASA through grant NGL 14-007-41 and by Northwestern University. The numerlcal computations were performed at the computing center of Northwestern University. 
TABLE 1

EVOLUTTON AT $\dot{\mathrm{M}}=5 \times 10^{-8} \mathrm{M}_{Q} \mathrm{yr}^{-1}$

\begin{tabular}{|c|c|c|c|c|c|c|c|}
\hline$t$ (years) & $4.72(5)$ & $1.27(6)$ & $2.24(6)$ & $2.40(6)$ & $2.40(6)$ & $2.40(6)$ & $2.40(6)$ \\
\hline $\log P_{\text {II }}$ & 4.608 & 4.981 & 5.239 & 5.326 & 5.288 & 5.221 & 5.003 \\
\hline $\log T_{m}$ & 7.713 & 7.853 & 7.942 & 7.985 & 8.140 & 8.230 & 8.404 \\
\hline$\Delta M\left(M_{\theta}\right)$ & $2.36(-2)$ & $\begin{array}{c}6.33(-2) \\
.\end{array}$ & $1.12(-1)$ & $1.20(-1)$ & $1.20(-1$ & $1.20(-1)$ & $1.20(-1)$ \\
\hline$\psi$ & 4.03 & 5.22 & 6.28 & .48 & 4.15 & 2.89 & 0.84 \\
\hline $\log \rho_{c}$ & 6.281 & 6.364 & 6.465 & 6.480 & 6.476 & 6.462 & 6.435 \\
\hline $\log \mathrm{T}_{\mathrm{c}}$ & 7.284 . & .7 .332$. & 7.393 & 7.402 & 7.400 & 7.391 & 7.374 \\
\hline $\mathrm{r}_{\text {nuc }}(8)$ & - & - & $4.00(14)$ & $3.76(12)$ & $4.57(7)$ & $4.36(5)$ & $1.56(3)$ \\
\hline$\varepsilon_{m}\left(\right.$ erg $\left.8^{-1} \mathrm{~s}^{-1}\right)$ & - & - & $1.83(1)$ & $2.14(3)$ & $2.52(8)$ & $3.25(10)$ & $1.36(13)$ \\
\hline
\end{tabular}

$T_{m}$ represents the maximum temperature in the helium-rich regton, $\rho_{m}$ represents the density at the mass point corresponding to $T_{m}$, and $\varepsilon_{\mathrm{m}}$ represents the maximum energy generation rate. 
TABLE 2

EVOLUTION AT $\dot{\mathrm{M}}=10^{-8} \mathrm{M}_{\theta} \mathrm{yr}^{-1}$

\begin{tabular}{|c|c|c|c|c|c|c|c|c|c|}
\hline$t($ years $)$ & $9.79(6)$. & $2.09(7)$ & $4.26(7)$ & $5.50(7)$ & $6.17(7)$ & $6.17(7)$ & $6.17(7)$ & $.6 .17(7)$ & $6.17(7)$ \\
\hline $108 P_{m}$ & 5.170 & 5.600 & 5.933 & 6.207 & 7.161 & 7.102 & 7.077 & 7.059 & 7.005 \\
\hline $\log \mathrm{T}_{\mathrm{m}}$ & 7.703 & 7.804 & 7.807 & 7.815 & .7 .866 & 8.024 & 8.321 & 8.518 & 8.795 \\
\hline$\Delta M\left(M_{\theta}\right)$ & $9.79(-2)$ & $2.09(-1)$ & $4.26(-1)$ & $5.50(-1)$ & $6.17(-1)$ & $6.17(-1)$ & $6.17(-1)$ & $6.17(-1)$ & $6.17(-1)$ \\
\hline$\psi$ & 7.88 & 14.88 & 23.69 & 33.75 & 96.15 & 62.71 & 30.70 & 19.02 & 9.31 \\
\hline $\log \rho_{c}$ & 6.443 & 6.685 & 7.186 & 7.502 & 7.689 & 7.689 & 7.689 & 7.689 & 7.689 \\
\hline $\log T_{c}$ & 7.439 & 7.723 & 7.783 & 7.825 & 7.856 & 7.856 & 7.856 & 7.856 & 7.856 \\
\hline$\tau_{\text {nuc }}(s)$ & - & - & - & - & $4.53(13)$ & $1.53(7)$ & $1.82(1)$ & $1.25(-2)$ & $7.67(-4)$ \\
\hline$\varepsilon_{m}\left(\operatorname{erg} g^{-1} g^{-1}\right)$ & - & - & - & - & $1.35(2)$ & $5.76(8)$ & $9.57(14)$ & $2.20(18)$ & $6.76(19)$ \\
\hline
\end{tabular}


TABLE 3

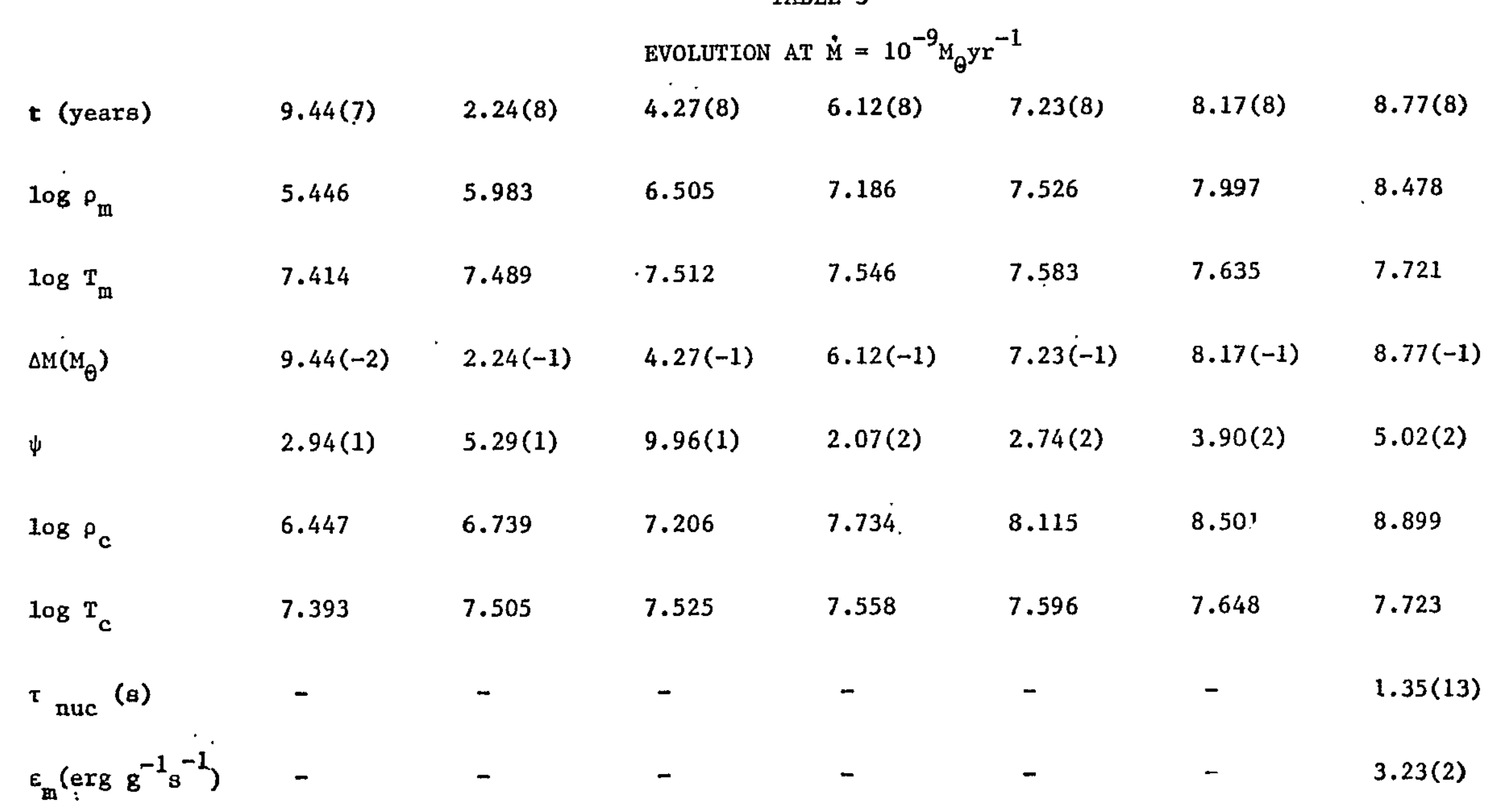


References

Arnett, W.D. 1969 ; Ap. and Space Sc1. , 5, 180.

Eggleton, P.P. 1971, M.N.R.A.S., 151, 351 . , 1972, ibtd., 156, 361.

Mazurek, T.J. 1973, Ap. and Space Sci., 23, 365.

Nariai, K., Nomoto, K., and Sugimoto, D. 1977, in Novae and Related

., $\ldots$ Stars, ed. M. Friedjung (D. Reidel Publ. Co., Dordrecht), p. 218.

Nomoto, K. and Sugimoto, D. 1977, Publ. Astron. Soc. Japan, 29, 765.

Paczynski, B. 1976, in Structure and Evolution of Close Binary Systems,

IAU Symp. 73, ed. P. Eggleton, S. Mitton, J. Whelan (D. Reidel

Publ: Co., Dordrecht), p. 75 .

Paczynski, B. and Zytkow, A. 1978, Ap.J., 222, 604.

Prialnik, D., Shara, M.M., and Shaviv, G. 1978, Astr. Ap., 61, 363.

Ritter, H. 1976, M.N.R.A.S., 175, 279.

Robinson, E.L. 1976, Ann. Rev. Astron. Astrophys., 14, 119.

Sion, E.M., Acierno, M.J., and Tomczyk, S. 1979, Ap.J., 230, 832.

Sparks, W.M., Starrfield, S.; and Truran, J.W. 1978, Ap. J., $220,1063$.

Starrfleld, S., Sparks, W.M., and Truran, J.W. 1974a, Ap.J. Supp., 28, 247.

Starrfield, S., Sparks, W.M., and Truran, J.W. 1974b, Ap.J.,. 192, 647.

Taam, R.E. 1977, Astrophys. Letters, 19, 47.

Taam, R.E. 1979, Ap. and Space Sci., in press.

Taam, R.E. and Faulkner, J. 1975, Ap.J., $198,435$.

Warner, B. 1976, in Structure and Evolution of Close Binary Systems,

IAU Symp. 73, ed. P: Eggleton, S. M1tton, J. Whelan (D. Reidel

Pub1. Co., Dordrecht), p. 85 .

Webbink, R.F. 1975, M.N.R.A.S., 171, 555.

Webbink, R.F., Truran, J.W., and Gallagheir, J.S., Bull. A.A.S., 10, 438. 
Figure Captions

Figure 1. Structural changes in the temperature density plane from the onset of mass accretion to the ignition of hellum for $\dot{\mathrm{M}}=5 \times 10^{-8} \mathrm{M}_{G} \mathrm{yr}^{-1}$. The time corresponding to each curve is given for clarity. The unlabelled curve corresponds to the distribution in the initial white dwarf model. "Vertical Iines" Indicate'the" location of the He-C composition Interface.

Figure 2. Same as for FIgure $I$ except $\dot{M}=10^{-8} \mathrm{M}_{G} \mathrm{yr}^{-1}$.

Figure 3. Same as for Figure 1 except $\dot{\mathrm{M}}=10^{-9} \mathrm{M}_{\mathrm{O}} \mathrm{yx}^{-1}$. 


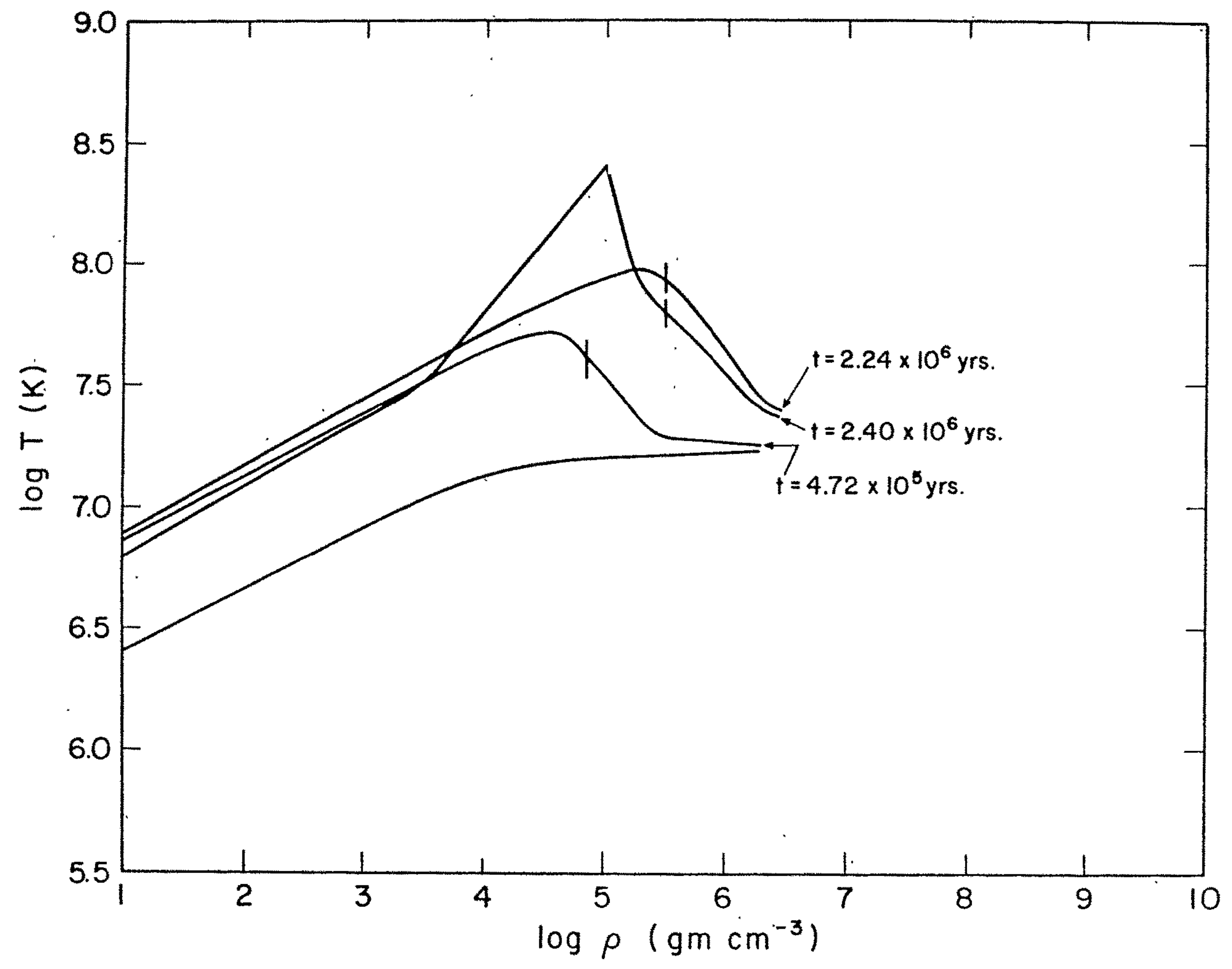




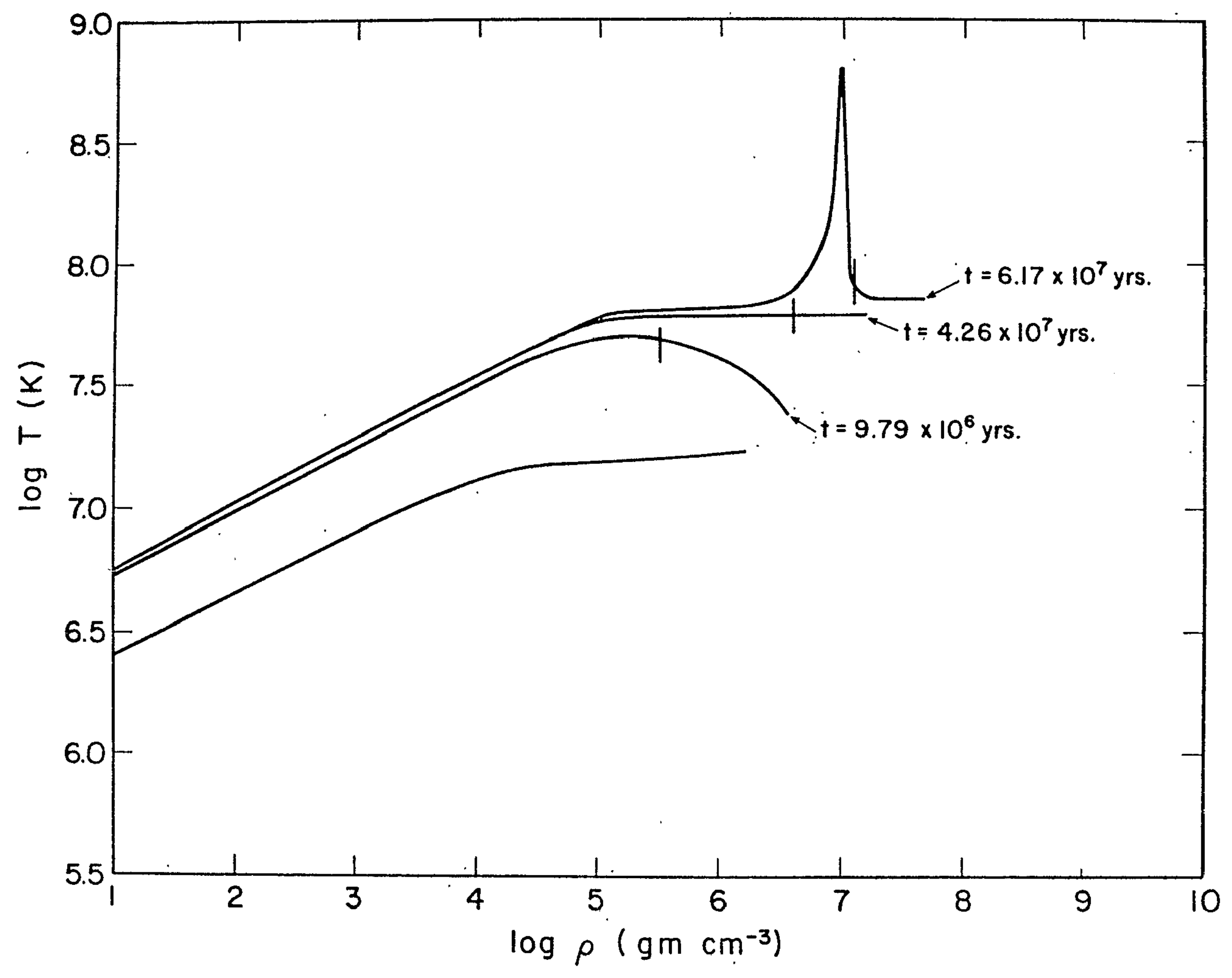




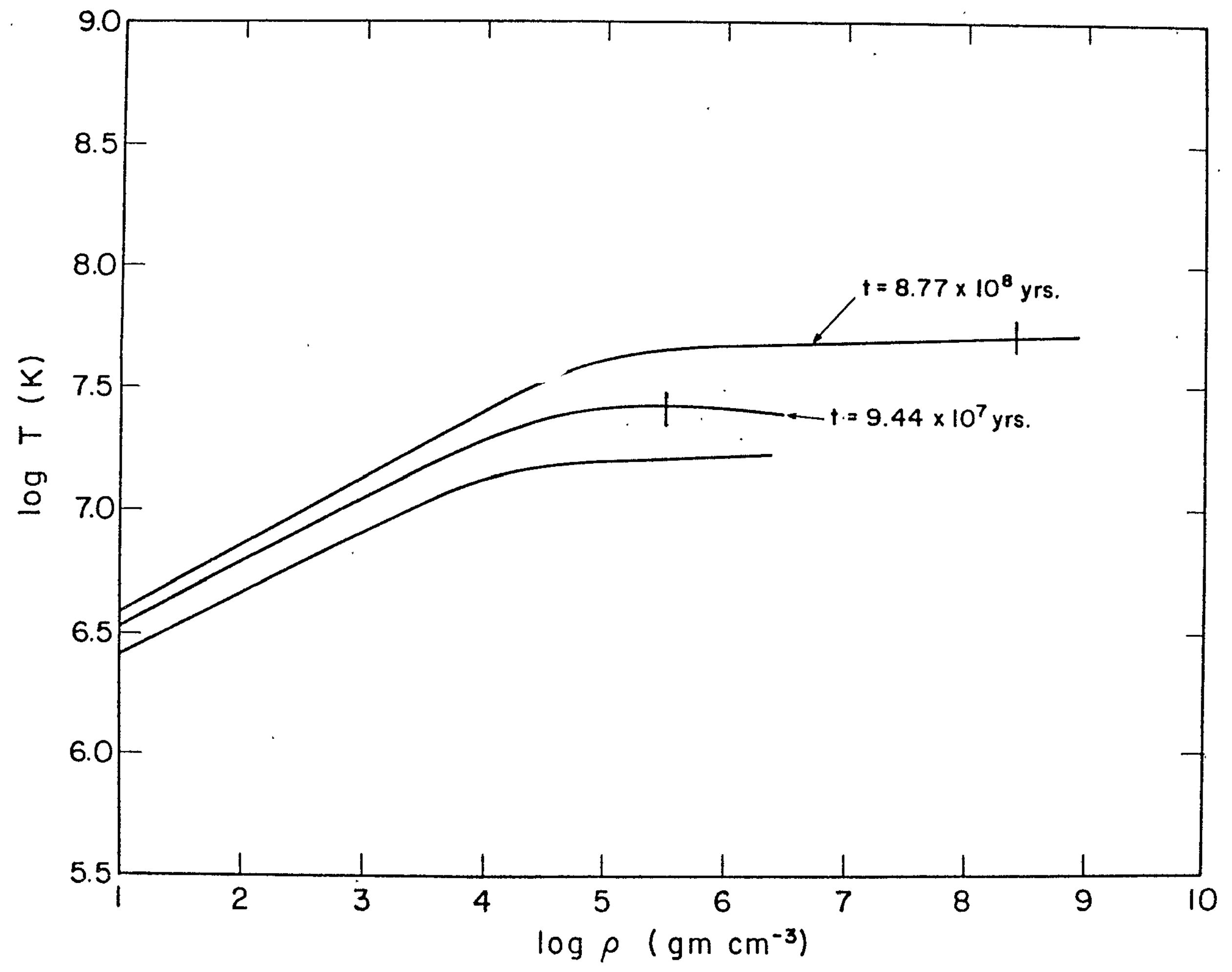

